\title{
Application of Caenorhabditis elegans (nematode) and Danio rerio embryo (zebrafish) as model systems to screen for developmental and reproductive toxicity of Piperazine compounds
}

\author{
Peter I. Racz ${ }^{\mathrm{a}, *, 1}$, Marjolein Wildwater ${ }^{\mathrm{b}, *, 1}$, Martijn Rooseboom ${ }^{\mathrm{c}}$, Engelien Kerkhof ${ }^{\mathrm{d}}$, \\ Raymond Pieters $^{\mathrm{b}, \mathrm{e}}$, Elena Santidrian Yebra-Pimentel ${ }^{\mathrm{a}}$, Ron P. Dirks ${ }^{\mathrm{a}}$, Herman P. Spaink ${ }^{\mathrm{f}}$, \\ Chantal Smulders ${ }^{c}$, Graham F. Whale ${ }^{g}$ \\ a ZF-screens BV, J.H. Oortweg 19, 2333 CH Leiden, The Netherlands \\ b University of Applied Sciences Utrecht, Heidelberglaan 7, 3584 CS Utrecht, The Netherlands \\ c Shell Health, Shell International B.V., Carel van Bylandtlaan 16, 2596 HR The Hague, The Netherlands \\ d University of Applied Sciences of Arnhem and Nijmegen, Laan van Scheut 2, 6525 EM Nijmegen, The Netherlands \\ e Institute for Risk Assessment Sciences, Yalelaan 104, 3584 CM Utrecht, The Netherlands \\ ${ }^{\mathrm{f}}$ Institute of Biology, Leiden University, Einsteinweg 55, 2333 CC Leiden, The Netherlands \\ ${ }^{g}$ Shell Health, Brabazon House, Threapwood Road, Manchester M22 ORR, United Kingdom
}

\section{A R T I C L E I N F O}

\section{Keywords:}

Piperazine

Zebrafish

Nematode

Danio rerio

Caenorhabditis elegans

Development

Reproduction

\begin{abstract}
A B S T R A C T
To enable selection of novel chemicals for new processes, there is a recognized need for alternative toxicity screening assays to assess potential risks to man and the environment. For human health hazard assessment these screening assays need to be translational to humans, have high throughput capability, and from an animal welfare perspective be harmonized with the principles of the 3Rs (Reduction, Refinement, Replacement).

In the area of toxicology a number of cell culture systems are available but while these have some predictive value, they are not ideally suited for the prediction of developmental and reproductive toxicology (DART). This is because they often lack biotransformation capacity, multicellular or multi- organ complexity, for example, the hypothalamus pituitary gonad (HPG) axis and the complete life cycle of whole organisms.

To try to overcome some of these limitations in this study, we have used Caenorhabditis elegans (nematode) and Danio rerio embryos (zebrafish) as alternative assays for DART hazard assessment of some candidate chemicals being considered for a new commercial application. Nematodes exposed to Piperazine and one of the analogs tested showed a slight delay in development compared to untreated animals but only at high concentrations and with Piperazine as the most sensitive compound. Total brood size of the nematodes was also reduced primarily by Piperazine and one of the analogs. In zebrafish Piperazine and analogs showed developmental delays. Malformations and mortality in individual fish were also scored. Significant malformations were most sensitively identified with Piperazine, significant mortality was only observed in Piperazine and only at the higest dose. Thus, Piperazine seemed the most toxic compound for both nematodes and zebrafish.

The results of the nematode and zebrafish studies were in alignment with data obtained from conventional mammalian toxicity studies indicating that these have potential as developmental toxicity screening systems. The results of these studies also provided reassurance that none of the Piperazines tested are likely to have any significant developmental and/or reproductive toxicity issues to humans when used in their commercial applications.
\end{abstract}

\section{Introduction}

New products that are brought to the market have to be proven safe for man and the environment. Hazard assessment of compounds, in close conjunction with exposure characteristics, are therefore essential and mandatory requirements. Accepted regulatory toxicity testing for chemicals currently requires mammalian studies (i.e. rat and rabbit), which are time- and money-consuming and increasingly considered

\footnotetext{
* Corresponding authors.

E-mail addresses: racz@zfscreens.com (P.I. Racz), marjolein.wildwater@hu.nl (M. Wildwater).

${ }^{1}$ These authors contributed equally, arranged on alphabetical order.
} 
unethical by society. Furthermore, especially when potential hazard for development and reproduction (DART) is considered, these mammalian test systems only show low predictive values to man (Sipes et al., 2011). Proper establishment of alternative testing strategies that are quick, low cost, ethical and predictive are therefore urgently required to reduce, refine and replace (3R principle) mammalian testing.

Historically the focus was set on the use of cell culturing systems to provide promising alternative testing strategies. While these systems have some benefits (e.g. the possibility of using human cells), these systems lack the complexity of a complete organism with different organs and cell-cell and tissue-tissue signaling, organismal defense mechanistic responses towards potential hazardous compounds and as such have their limitations in possible applicability. There is a need for lower cost, more rapid, less animal intensive studies to help screen potential new products to identify those which raise concerns and may require additional assessment. Such tests could also have value to help in the definition of existing product categories under the EU REACH regulations by either 'proving' similar modes of actions and/or identifying products with the highest potential to cause adverse developmental/reproductive effects for longer term animal tests.

Recently two alternative in vivo model systems, Caenorhabditis elegans (nematode) and Danio rerio (zebrafish) which were well known and used in the field of Developmental and Molecular Biology became noticed as potential promising test systems for hazard assessment (Avila et al., 2012; Ballatori, 2002; Boyd et al., 2016, 2010; Brannen et al., 2010; Hermsen et al., 2011; Leung et al., 2008; Panzica-Kelly et al., 2010; Dhawan et al., 1999; Selderslaghs et al., 2009, 2012). Both species share high genetic homology to man $(\sim 60 \%$ for nematodes and $70 \%$ for zebrafish), show cell biologically conserved molecular responses (like organ development, cell and tissue signaling etc.) and have proven their translational value (for example, the Nobel prize for the discovery of apoptosis and miRNAs was rewarded to nematode researchers (Fire et al., 1998) and both systems are commonly used in medical research (Ordas et al., 2015; Phillips and Westerfield, 2014; Poureetezadi and Wingert, 2013; Stewart et al., 2014).

Both nematodes and zebrafish embryos until 5-day post-fertilization (5dpf) are not considered animals according to relevant animal welfare acts and regulations. As nematodes and zebrafish are optically transparent small animals with a high reproductive and developmental turnover they can be considered as an alternative test species for DART assessment. Because of the high number of progeny each nematode is able to produce around 250 eggs within 3 days, and one zebrafish animal can produce up to 300 eggs in a week, these organisms have the potential for high throughput screening. Nematode progeny is furthermore genetically tractable as nematodes are self-fertilizing hermaphrodites of only $1 \mathrm{~mm}$ in size that have shown highly reproducible predictive developmental timing (Sulston and Horvitz, 1977; Sulston et al., 1983). Young nematode larvae develop within 3 days to reproductive hermaphrodites. In zebrafish, development is also rapid as most organs are formed during early embryo development within 3 days post fertilization. Thus, these systems show high potential to be properly validated as alternative 3R DART test systems.

In the research project, CRACKIT PreDART funded by the NC3Rs (UK's national organisation which leads the discovery and application of new technologies and approaches for 3R purposes), the methodology for implementation of nematodes and zebrafish as alternative 3R test models for developmental and reproductive toxicity was set up (publications in progress). Out of 31 well characterized DART compounds tested in nematodes and zebrafish, respectively 27 and 23 were properly predictive for DART. Interestingly, the ones that were missed by one of the two systems were picked up as DART compounds by the other system and thus all compounds were scored correctly by combinatorial testing using nematodes and zebrafish.

In this study a number of Piperazine analogs for commercial application have been evaluated in an experimental screen for reproductive and developmental toxicity using nematodes and zebrafish embryos. The screening studies are being evaluated for their potential to detect developmental toxicity (e.g. intrauterine death including preimplantation loss, structural abnormalities, altered growth and functional deficits) while avoiding significant use of animals.

In these initial investigations, compounds were tested to assess if the 'screening' studies could detect differences in their potential to cause developmental/reproductive effects. The amines selected were Piperazine (CAS: 110-85-0) and the Piperazine analogs A, B and-C. (PIP-A; PIP-B and PIP-C) One advantage of these substances was that these are stable and water soluble thereby mitigating any concerns regarding their exposure to the organisms.

Piperazine has been classified as a category 2 repro-toxicant under the EU's Classification, Labelling and Packaging (CLP) regulations (EC) No $1272 / 2008$ and was used as a positive control in the studies described, whereas the Piperazine analogs have not been tested and currently have not been classified. In rodents Piperazine is a weak class-2 toxicant as it causes embryotoxic effects as resorptions, retardation of ossification, reduced foetal weights and malformations only at high doses. These effects are considered to be a secondary effect of maternal toxicity, rather than a direct developmental or reproductive toxicity effect (Cross et al., 1954; Ridgway, 1987; Risk et al., 2005).

\section{Materials \& methods}

\subsection{Materials}

Piperazine (95\% purity) was obtained from Sigma-Aldrich (P45907), Piperazine analogs (95\% purity) where provided by Shell.

\subsection{Nematodes}

Nematodes of the N2 strain were synchronized using hypochlorite and hatched L1 larvae were exposed to the compound that was dissolved in nematode growth medium (NGM). L1 larvae were allowed to develop into adults and subsequently transferred daily to fresh medium. The range of exposure concentration was the same for all compounds, i.e. $10^{-7} \mathrm{M}, 10^{-6} \mathrm{M}, 10^{-5} \mathrm{M}, 10^{-4} \mathrm{M}, 10^{-3} \mathrm{M}, 10^{-2} \mathrm{M}$. Brood size was determined by daily passage of adult nematodes onto new plates and subsequent counting of offspring. The sum of all progeny on all subsequent wells was used to calculate the total brood size per nematode. Developmental progression was scored by analyzing stage-specific parameters (organ development rate) as shown in Fig. 1, Fig. 2, Table 1, Tables S2 and S3 using the published cell lineage papers (Sulston and Horvitz, 1977; Sulston et al., 1983). Note: Control populations should never show any deviation in developmental progression (developmental delay). If they do, experiments are aborted.

Four days before the start of the experiment, nematodes are grown to bulk quantities on normal food and media (20 times a $5 \mathrm{~cm}$ NGM plate with bacterial OP50 food) to ensure sufficient animals to enable the compound test assay. One day before the start of the experiment (the start of exposure), these nematode cultures were bleached to synchronize progeny for the assay. In the absence of food bleaching results in a synchronous population of L1 staged animals ready for the test the next day.

On the first day of the test (day 0) hatched L1 larvae were placed onto the NGM agar containing compound and grown at $15{ }^{\circ} \mathrm{C}$ for $72 \mathrm{~h}$ to become L4 larvae. Then they were checked under the microscope for developmental age and morphological effects as listed in Table 1.

Additionally, reproduction effects were scored by exposing 30 individual L4 animals in three 12 wells plates. For a period of 4 additional days, these nematodes were transferred each day to a new well leaving any progeny left on the old plate to grow for one more day before counting and assessing the viability of the progeny (hatched eggs) and total brood size.

Proper development of the offspring was assessed by examining them under a Zeiss Axio Imager M2. The nematode cell lineage is 


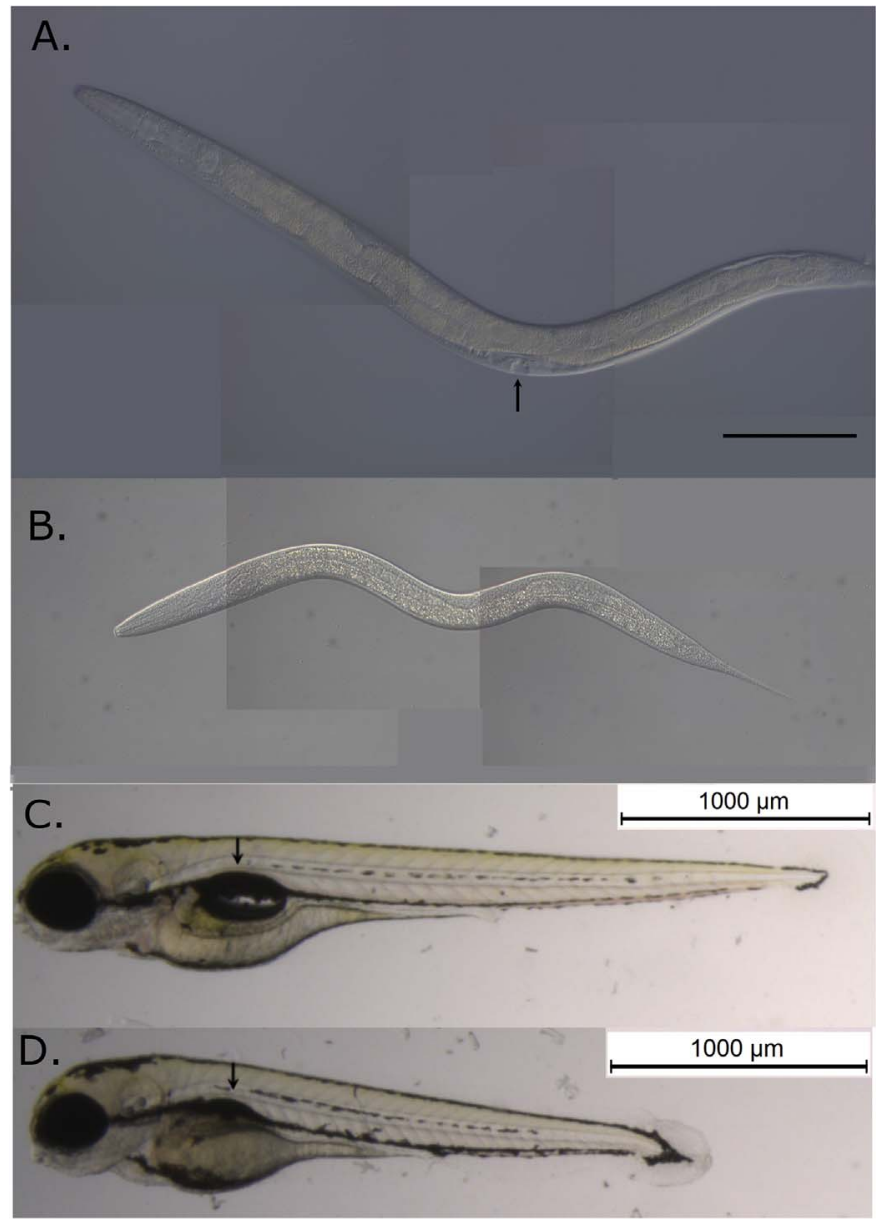

Fig. 1. Phenotypic effects in nematodes and zebrafish exposed to Piperazine reveal mild effects in development.

Nematodes and zebrafish both show a mild developmental delay when exposed to high concentrations of Piperazine. While $100 \%$ of the nematode larvae developed to L4 stage in the control after $25 \mathrm{~h}$ exposure at $20^{\circ} \mathrm{C}$ (A), $10^{-2} \mathrm{M}$ Piperazine exposure revealed $71 \%$ L3 and 29\% L2 stage animals (B) (also shown in Table S2). Fig. B shows an L3 stage animal. The arrow in A indicates the vulva, a developmental marking point of L4 stage. Scalebar: $100 \mu \mathrm{m}$. Panel C and D show zebrafish embryos and show delayed zebrafish development (D) compared to the control (C). In zebrafish in $60 \%$ of the cases the swim bladder appeared undeveloped at $4 \mathrm{dpf}$ after Piperazine exposure, indicative for developmental delay effects (position of swim bladder is indicated by an arrow). Also tail length and head-trunk angle are affected in the animal in D and are indicative for developmental delay.

completely mapped and development is traceable within precision of hours using the state of organ development as a reference point in relation to the total developmental time (development of young larvae only starts when they receive food; $t=0$ ). Both vulva development as well as gonadogenesis can be scored in L4 larvae to monitor developmental progression. Clear synchronous stages of the developing vulva can be observed during time, like early divisions of the vulva precursor cells (VPCs) in L3 stage of development (starting after $29 \mathrm{~h}$ at $20^{\circ} \mathrm{C}$ ), appearance of the initial vulva cleft $\left(34 \mathrm{~h}\right.$ at $\left.20^{\circ} \mathrm{C}\right)$, Christmas tree $(40 \mathrm{~h}$ at $20^{\circ} \mathrm{C}$ ), and vulva lip formation $\left(50 \mathrm{~h}\right.$ at $20^{\circ} \mathrm{C}$ ). In case of developmental delay, all parameters should have the features that are representative for younger animals than expected according to experimental duration. Because of these easy set of scorable characteristics, affected development can be monitored in a precise manner and can be separated from organ specific effects.

\subsection{Zebrafish}

Zebrafish experimental procedures were conducted in accordance with local and international regulations and followed the guidelines on the protection of experimental animals by the Council of Europe, Directive 2010/63/EU reduction, replacement and refinement strategy. Zebrafish were handled and maintained according to standard protocols ("The Zebrafish Model Organism Database," ZFIN www.zfin.org). Zebrafish larvae were collected from laboratory cultures. All tests were undertaken at $28{ }^{\circ} \mathrm{C}$ under a $14 \mathrm{~h}: 10 \mathrm{~h}$ dark-light cycle. Controls and tests solutions were prepared in 'egg water' $\left(60 \mu \mathrm{g} / \mathrm{ml}\right.$ Instant Ocean $^{\mathrm{TM}}$ sea salt, Sera Marin in distilled water). Individual larvae were raised in a separate well in a 24 well polypropylene plates containing $2 \mathrm{ml}$ of the test substance $\left(10^{-7} \mathrm{M}, 10^{-6} \mathrm{M}, 10^{-5} \mathrm{M}, 10^{-4} \mathrm{M}, 10^{-3} \mathrm{M}, 10^{-2} \mathrm{M}\right)$. Larvae were exposed in the static way. For phenotypic observation bright-field, Leica M165C stereomicroscope was used at various magnification $(2 \times-16 \times)$ equipped with a DFC420C digital colour camera (Leica Microsystems).

20 newly fertilized zebrafish eggs were selected per replicate, between 2 and 64 cell stage (before blastulation) and exposed to test chemicals for a period of $96 \mathrm{~h}$. The development of the embryos was followed on a daily basis. After $96 \mathrm{~h}$, lethality was assessed on the basis of either/or: (I) coagulation of fertilized eggs, (II) lack of somite formation, (III) lack of heartbeat. At the end of the $96 \mathrm{~h}$ exposure period, fish larvae behavior in response to mechanical stimuli and phenotypic changes were recorded. Unresponsive behavior of the test is indicative of abnormal development or destruction of the nervous system and/or abnormality of the muscle contraction. Phenotypic examinations were undertaken on 20 larvae per concentration using relevant endpoints identified during the NC3R Crack it PREDART project (Table 1 and Table S4). The procedure was performed in duplicate. $10 \%$ deviation from zero incidents was accepted for the internal control fish (4 per 24 well plate) similarly to what has been agreed as acceptable in the Fish Embryo Acute Toxicity (FET) Test (OECD/OCDE 236). The phenotypic assessments, which were considered to be indicative of teratogenicity, included observation of abnormalities in organ development (Table 1). Acute toxicity (lethality) and delayed development were also scored on day 4 post fertilization. Spontaneous incidents in the untreated control group were scored as well. In the case of low occurrence $(<10 \%)$ in the experiment, the results were normalized to the untreated control group and the score of the spontaneous events were subtracted from the result. In the case of higher percentage of spontaneous death or malformation in the control group ( $>10 \%$ ), the test became invalid and was discarded. Characterization of normal development of the embryo was followed in the untreated control group and was identified based on the standard developmental timeline (Kimmel et al., 1995). Developmental delay was based on three main phenotypic appearances: head-trunk angle, tail length and occurrence of the swim bladder. When effects in at least two characteristics were scored, this was indicated as developmental delay. Note: delayed development might be a secondary effect of abnormal organ development and conclusions regarding developmental delay should, therefore, be treated with caution.

\section{Results \& discussion}

Nematodes and zebrafish larvae were exposed to a range of concentrations of Piperazine and three Piperazine analogs. Developmental effects were scored by analyzing organ development and a set of other parameters (see Table 1 and Tables S2 and S4).

No chemical analysis was undertaken to assess exposure concentrations. However, based on their physicochemical properties, including water solubility, all the compounds are expected to be soluble and well absorbed (Lipinski, 2004). Furthermore, as Piperazine appears to be well absorbed (with peak plasma concentrations attained $1 \mathrm{~h}$ after oral administration according to the REACH dossier), it can be assumed that nematodes and zebrafish have been exposed significantly systemically.

Nematodes exposed to Piperazine and PIP-A, showed a slight delay in development compared to untreated animals but only at high 
A.

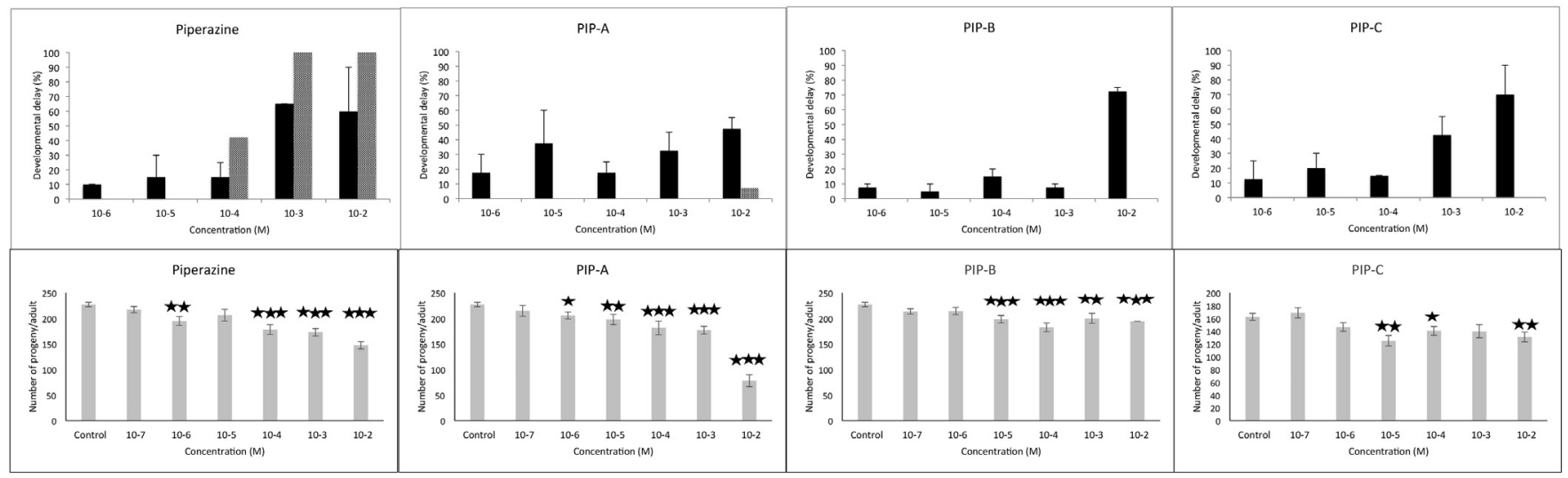

Fig. 2. Only high concentrations of Piperazines affect developmental rate and reproduction in nematodes and zebrafish.

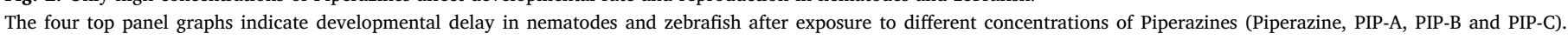

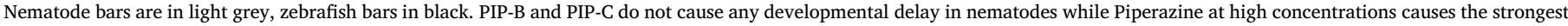

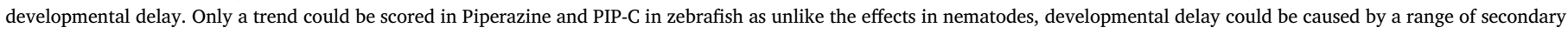

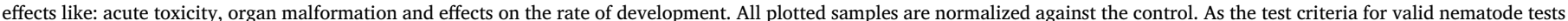

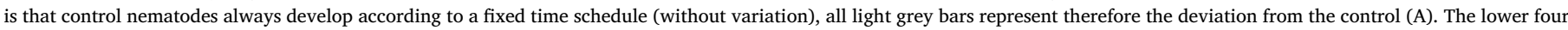

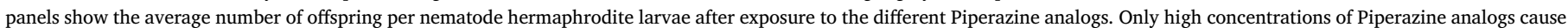

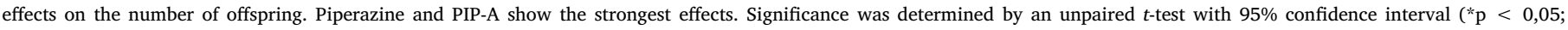
$\left.{ }^{* *} \mathrm{p}<0,01,{ }^{* * *} \mathrm{p}<0,001\right)(\mathrm{B})$. Error bars are indicating the standard error (standard deviation $/ \sqrt{ } \mathrm{n}$ ).

concentrations (starting at $10^{-4} \mathrm{M}$ and $10^{-2} \mathrm{M}$, respectively), whereas PIP-B and PIP-C did not show any delay effects (Figs. 1 and 2 and Table S2). Total brood size of the nematodes was also reduced when exposed to Piperazine and PIP-A at $10^{-6} \mathrm{M}$ and higher, for PIP-B and PIP-C at
$10^{-5} \mathrm{M}$ and higher (Fig. 2B and Table S3). There was no effect on larval mortality in nematodes after any of the treatments.

In zebrafish an increased dose of Piperazine and analogs indicated an increase in the percentage of fish with developmental delay.

Table 1

Scoring table of potentially affected organ development \& reproduction.

A broad range of developmental and reproductive effects were scored after Piperazine exposure in nematodes and zebrafish. Both species were only mildly affected by the Piperazines.

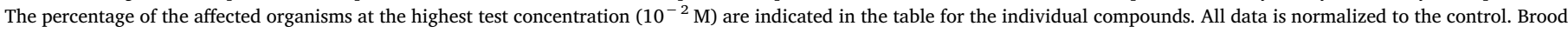

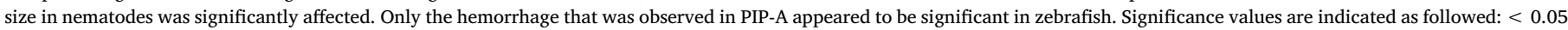
$(*),<0.01(* *)$ and $<0.001\left(^{* * *}\right)$. The number of incidences and statistics can be found in Tables S2, S3, S4 and S5.

\begin{tabular}{|c|c|c|c|c|c|c|}
\hline \multirow[t]{2}{*}{ Organism } & \multirow[t]{2}{*}{ Phenotype } & \multirow[t]{2}{*}{ Effect } & \multicolumn{4}{|c|}{ Affected organisms (\%) at highest test concentration $\left(10^{-2} \mathrm{M}\right)$} \\
\hline & & & Piperazine & PIP-A & PIP-B & PIP-C \\
\hline $\mathrm{N}$ & $\begin{array}{l}\text { Reproductive organs (gonad, } \\
\text { vulva) }\end{array}$ & $\begin{array}{l}\text { Organisation, shape, size and absence of the organs; } \\
\text { multi vulva }\end{array}$ & 0 & 0 & 0 & 0 \\
\hline $\mathrm{N}$ & Nervous system & Movement, egg laying, behavoir & 0 & 0 & 0 & 0 \\
\hline $\mathrm{N}$ & Intestine & Organisation, shape, size and presence of the organs & 0 & 0 & 0 & 0 \\
\hline $\mathrm{N}$ & Cuticle & $\begin{array}{l}\text { Molting problems, protruding/burst through vulva, } \\
\text { dumpy, blistered }\end{array}$ & 0 & 0 & 0 & 0 \\
\hline $\mathrm{N}$ & Muscles & Movement, egg laying, uncoordinated movements & 0 & 0 & 0 & 0 \\
\hline ZF & Fin & & 0 & 0 & 0 & 0 \\
\hline \multirow[t]{4}{*}{ ZF } & Heart & Acardia - absence of heart & 0 & 0 & 0 & 0 \\
\hline & & Pericardial oedema & 7.5 & 10.0 & 2.5 & 0 \\
\hline & & Tube heart formation (heart has no chamber) & 0 & 0 & 0 & 0 \\
\hline & & Cardiac enlargement & 0 & 0 & 0 & 0 \\
\hline \multirow[t]{3}{*}{ ZF } & Brain (head) & Brachycephalic (short broad head) & 0 & 0 & 0 & 0 \\
\hline & & Dolichocephalic (long narrow head) & 0 & 0 & 0 & 0 \\
\hline & & Reduced development the nose and the jaw & 0 & 0 & 5 & 0 \\
\hline $\mathrm{ZF}$ & Spine & Bent tail, bent head-trunk angle & 0 & 0 & 2.5 & 2.5 \\
\hline \multirow[t]{2}{*}{$\mathrm{ZF}$} & Eye & Cyclopia (one eye) & 0 & 0 & 0 & 0 \\
\hline & & Eye oedema & 0 & 0 & 0 & 0 \\
\hline $\mathrm{N}$ & Clear & Often correlated to defects in FGF signaling pathway & 0 & 0 & 0 & 0 \\
\hline $\mathrm{N}$ & Chromosomal instability & High incidence of males & 0 & 0 & 0 & 0 \\
\hline $\mathrm{N}$ & Variably abnormal & $\begin{array}{l}\text { Often correlated with cell-cell contact problems in } \\
\text { epithelial cells }\end{array}$ & 0 & 0 & 0 & 0 \\
\hline $\mathrm{N}$ & Size & Often correlated with cell division problems & 0 & 0 & 0 & 0 \\
\hline $\mathrm{N}$ & Reduced number of progeny & Percentage & $35.1 * * *$ & $65.7 * * *$ & $14.6^{* * *}$ & $19.3^{* *}$ \\
\hline $\mathrm{N}$ & Dauers & $\begin{array}{l}\text { Often correlated with problems in metabolism or eating } \\
\text { problems }\end{array}$ & 0 & 0 & 0 & 0 \\
\hline $\mathrm{ZF}$ & Hemorrhage & Blood collection in abnormal places & 0 & $10 * * *$ & 2.5 & 2.5 \\
\hline $\mathrm{ZF}$ & Larvae movement & Partial hatch/no reaction to touch stimulus & 0 & 0 & 0 & 0 \\
\hline $\mathrm{ZF}$ & Excessive opercular movement & (Absence of oxygen) & 0 & 0 & 0 & 0 \\
\hline ZF & Abnormal hatching & & 0 & 0 & 2.5 & 0 \\
\hline \multirow[t]{2}{*}{ ZF } & Pigment formation & Abnormal pattern & 2.5 & 2.5 & 0 & 0 \\
\hline & & Absence of pigmentation & 0 & 0 & 0 & 0 \\
\hline
\end{tabular}


PIP

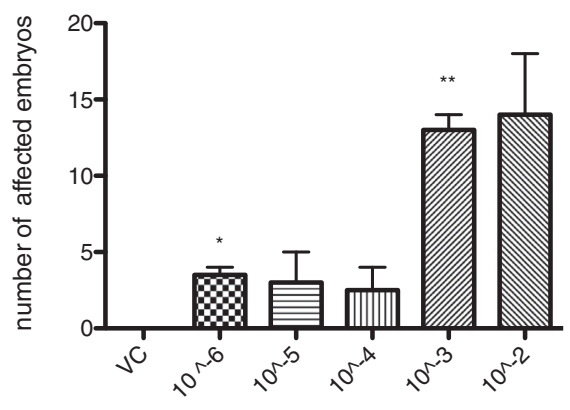

Concentration [M]

PIP-B

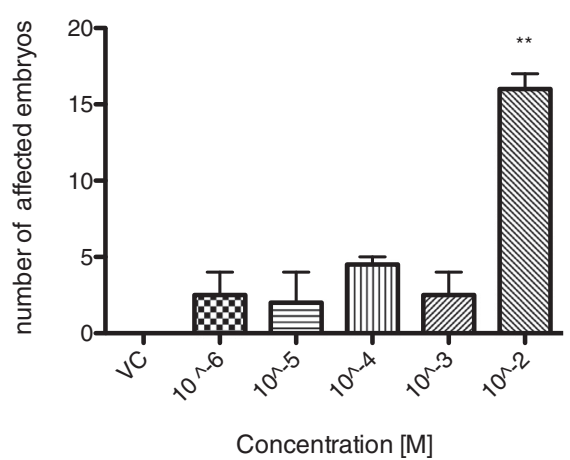

PIP-A

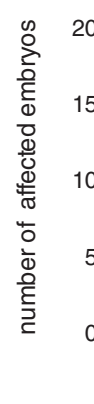

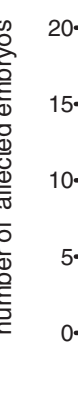

$$
\text { (1) }
$$
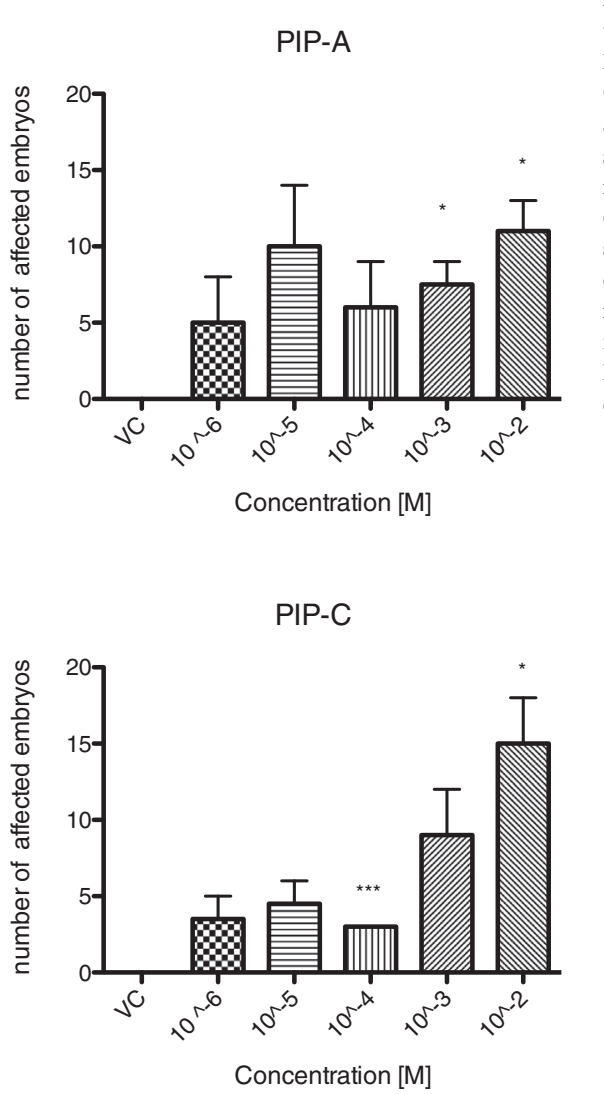

Fig. 3. The number of affected zebrafish embryos upon treatment with Piperazine and its analogos.

Malformation occurrence was registered based on a CrackIT scoring table (y-axis represents the number of affected embryos). The bars represent the mean and standard deviation of the experiments $(n=2$ experiments). The malformation occurrence was normalized to controls, and acute toxicity effects were corrected. A significant number of fish with malformations can be observed at Piperazine concentrations of $10^{-6} \mathrm{M}$. The number of incidences increases at higher concentrations in all analogs (see also Tables S4 and S5). Statistical values were calculated with an unpaired $t$-test with $95 \%$ confidence interval $\quad\left({ }^{*} \mathrm{p}<0,05 ; \quad * * \mathrm{p}<0,01\right.$, $* * * \mathrm{p}<0,001)$.
Developmental delay could occur as secondary effects of malformations or acute toxicity and thus possibly represents an accumulative effect.

Malformations in individual fish were therefore also scored (Fig. 3 and Table S4). A significant number of fish with malformations could be seen at Piperazine concentrations of $10^{-6} \mathrm{M}$. Yet, a huge increase in the number of incidences took only place at higher concentrations in all analogs (Fig. 3, Table S4, Table S5). PIP-A and PIP-B required an even $1000 \times$ higher dose before malformations were statistically relevant. Thus, Piperazine seemed the most toxic compound in zebrafish with only high increase in incidence numbers at high dose.

None of the tested compounds showed effects on heart function in zebrafish (bradycardia, tachycardia and arrhythmia). In addition, there was no indication of neurological functional defects as all of the exposed zebrafish larvae, even in the highest concentrations, responded to the touch stimulus test (Table 1, data not shown). A very weak effect was found when mortality was assessed in the highest concentration of Piperazine (Table S1).

In summary, from the Piperazines tested in the current study, Piperazine itself was the most potent toxicant to induce both reproduction toxicity and developmental delay in nematodes and malformations and mortality in zebrafish. Piperazine and PIP-A showed the highest sensitivity in affecting brood size in nematodes $\left(10^{-6} \mathrm{M}\right)$. These results are in alignment with the reported test data for rats and rabbits on Piperazine, where indications of reproductive effects were observed at high test concentrations (Cross et al., 1954; Ridgway, 1987; Risk et al., 2005). Furthermore, the observed responses are considered to be a consequence of maternal toxicity rather than a direct developmental or reproductive effect per se. Therefore, based on all of the above it is concluded that the Piperazine analogs tested are unlikely to be developmental toxicants.

An important consideration from the outset was the speed and cost of the alternatives in comparison to longer term 'traditional' DART studies. The tests have been compared in Table 2.

These data demonstrates that in comparison to the conventional
Table 2

Overview of different test methodologies for assessment of developmental and reproduction toxicity.

Golden standard OECD protocols are compared with 3R nematodes and zebrafish test models. These latter two models show that testing is fast, low cost and 3R proof.

\begin{tabular}{|c|c|c|c|c|}
\hline & Nematode & Zebrafish & OECD 414 & $\begin{array}{l}\text { OECD 416/ } \\
443\end{array}$ \\
\hline Indicative cost & Low & Low & Moderate & High \\
\hline Study duration & 1 week & 1 week & 3 weeks $^{\mathrm{b}}$ & $\begin{array}{l}30 \text { weeks }^{\mathrm{b}} / \\
21 \text { weeks }^{\mathrm{b}}\end{array}$ \\
\hline Exposure & Buffer & Water & Gavage & Gavage \\
\hline 3Rs issues & None & Vertebrate & Rats \& rabbits & Rats \\
\hline $\begin{array}{l}\text { Number of } \\
\text { animals used }\end{array}$ & None & $\begin{array}{l}\text { None (until } \\
5 \mathrm{dpf})\end{array}$ & $\begin{array}{l}\sim 900 \text { rats, } \\
\sim 500 \text { rabbits }\end{array}$ & $\begin{array}{l}\sim 2600 / \\
1400^{\mathrm{a}} \text { rats }\end{array}$ \\
\hline $\begin{array}{l}\text { Regulatory } \\
\text { acceptability }\end{array}$ & $\begin{array}{l}\text { No (screen } \\
\text { WoE) }\end{array}$ & $\begin{array}{l}\text { No (screen } \\
\text { WoE) }\end{array}$ & Yes & Yes \\
\hline
\end{tabular}

a Basic design, i.e. no cohorts and extension to F2.

${ }^{\mathrm{b}}$ In-life portion of the study.

DART studies the alternative methods are rapid, far less time consuming and could significantly reduce animal use. At the moment nematodes and zebrafish tests are not yet suitable to make translational statements on effective concentration levels in higher systems nor in other aspects of risk assessment. This study indicates however that there is a relevance to use these assays as alternative screening tests to identify potential developmental and reproductive toxicity effects of compounds early in the product developmental pipeline.

Further evidence of the value of these assays comes from NC3Rs CrackIT PREDART challenge project in which a DART hazard assessment of a whole group of 31 well-known positives was analyzed using Dictyostelium discoideum (slime mould), nematodes and zebrafish embryos. For a selected group of compounds, the molecular response of the three different species were assessed using RNAseq analyses. Despite the fact that different phenotypic outcomes were observed, the 
toxicogenomic profile identified potential molecular mechanisms with human relevance and was shared across the test species (https://www. nc3rs.org.uk/integrative-dictyostelium-c-elegans-and-zebrafishapproach-assess-dart, manuscripts in prep).

\section{Conclusions}

The fact that the results of the nematodes and zebrafish assays are in alignment with data obtained from mammalian toxicity studies indicate that these have potential as developmental and reproductive toxicity screens without the need to use significant numbers of animals. The results of these studies also provide indication that none of the Piperazine analogs tested are likely to have any significant developmental issues to humans when used in commercial applications.

Supplementary data to this article can be found online at http://dx. doi.org/10.1016/j.tiv.2017.06.002.

\section{Funding sources}

This work was supported by Shell.

\section{Transparency document}

The http://dx.doi.org/10.1016/j.tiv.2017.06.002 associated with this article can be found, in the online version.

\section{Acknowledgements}

We would like to acknowledge Eleanor Michie for artwork and the NC3Rs for supporting the technology establishment that was used to address the research questions in this research in the CrackIT project PREDART (project number 25432-175153).

\section{References}

Avila, D., Helmcke, K., Aschner, M., 2012. The Caenorhabiditis elegans model as a reliable tool in neurotoxicology. Hum. Exp. Toxicol. 31, 236-243. http://dx.doi.org/10. $1177 / 0960327110392084$

Ballatori, N., 2002. Defining the molecular and cellular basis of toxicity using comparative models. Toxicol. Appl. Pharmacol. 183, 207-220. http://dx.doi.org/10.1006/ taap.2002.9488.

Boyd, W.A., McBride, S.J., Rice, J.R., Snyder, D.W., Freedman, J.H., 2010. A highthroughput method for assessing chemical toxicity using a Caenorhabditis elegans reproduction assay. Toxicol. Appl. Pharmacol. 245, 153-159. http://dx.doi.org/10. 1016/j.taap.2010.02.014.

Boyd, W.A., Smith, M.V., Co, C.A., Pirone, J.R., Rice, J.R., Shockley, K.R., Freedman, J.H., 2016. Developmental effects of the ToxCast ${ }^{\mathrm{TM}}$ phase I and phase II chemicals in Caenorhabditis elegans and corresponding responses in zebrafish, rats, and rabbits. Environ. Health Perspect. 124, 586-593. http://dx.doi.org/10.1289/ehp.1409645.

Brannen, K.C., Panzica-Kelly, J.M., Danberry, T.L., Augustine-Rauch, K.A., 2010.

Development of a zebrafish embryo teratogenicity assay and quantitative prediction model. Birth Defects Res. B Dev. Reprod. Toxicol. 89, 66-77. http://dx.doi.org/10. 1002/bdrb.20223.

Cross, B.G., David, A., Vallance, D.K., 1954. Piperazine adipate: a new anthelmintic agent: part II. Toxicological and pharmacological studies. J. Pharm. Pharmacol. 6, 711-717. http://dx.doi.org/10.1111/j.2042-7158.1954.tb11006.x.

Dhawan, R., Dusenbery, D.B., Williams, P.L., 1999. Comparison of lethality, reproduction, and behavior as toxicological endpoints in the nematode Caenorhabditis elegans. J. Toxicol. Environ. Health 58 (7), 451-462.

Fire, Andrew, Xu, SiQun, Montgomery, Mary, Kostas, Steven A., Driver, Samuel E., Mello, Craig C., 1998. Potent and specific genetic interference by double-stranded RNA in Caenorhabditis elegans. Nature 391, 806-811. http://dx.doi.org/10.1038/35888.

Hermsen, S.A.B., Pronk, T.E., van den Brandhof, E.J., van der Ven, L.T.M., Piersma, A.H., 2011. Chemical class-specific gene expression changes in the zebrafish embryo after exposure to glycol ether alkoxy acids and 1,2,4-triazole antifungals. Reprod. Toxicol. 32, 245-252. http://dx.doi.org/10.1016/j.reprotox.2011.05.010.

Kimmel, C.B., Ballard, W.W., Kimmel, S.R., Ullmann, B., Schilling, T.F., 1995. Stages of embryonic development of the zebrafish. Dev. Dyn. 203, 253-310.

Leung, M.C., Williams, P.L., Benedetto, A., Au, C., Helmcke, K.J., Aschner, M., Meyer, J.N., 2008. Caenorhabditis elegans: an emerging model in biomedical and environmental toxicology. Toxicol. Sci. 106 (1), 5-28. http://dx.doi.org/10.1093/toxsci/ kfn121.

Lipinski, C.A., 2004. Lead- and drug-like compounds: the rule-of-five revolution. Drug Discov. Today Technol. 1, 337-341. http://dx.doi.org/10.1016/j.ddtec.2004.11.007.

Ordas, A., Raterink, R., Cunningham, F., Jansen, H.J., Wiweger, M.I., Jong-raadsen, S., 2015. Testing tuberculosis drug efficacy in a zebrafish high-throughput. Antimicrob. Agents Chemother. 59 (2), 753-762. http://dx.doi.org/10.1128/AAC.03588-14.

Panzica-Kelly, J.M., Zhang, C.X., Danberry, T.L., Flood, A., DeLan, J.W., Brannen, K.C., Augustine-Rauch, K.A., 2010. Morphological score assignment guidelines for the dechorionated zebrafish teratogenicity assay. Birth Defects Res. B Dev. Reprod. Toxicol. 89, 382-395. http://dx.doi.org/10.1002/bdrb.20260.

Phillips, J.B., Westerfield, M., 2014. Zebrafish models in translational research: tipping the scales toward advancements in human health. Dis. Model. Mech. 7 (7), 739-743. http://dx.doi.org/10.1242/dmm.015545.

Poureetezadi, S.J., Wingert, R.A., 2013. Congenital and Acute kidney disease:translational research insights from zebrafish chemical genetics. Gend. Med. 1 (3), 112. http://dx.doi.org/10.4172/2327-5146.1000112.

Ridgway, P., 1987. Piperazine phosphate. Rat teratology study. In: Rep. to Reckitt Coleman from Toxicol Lab. Ltd. Ledbuty, Herefordshire, (https://www.echa.europa. eu/web/guest/registration-dossier/-/registered-dossier/14941).

Risk, V., Of, A., li, C., Pentahydrate, S., Trihydroxide, D.C., 2005. European Union Risk Assessment Report.

Selderslaghs, I.W.T., Van Rompay, A.R., De Coen, W., Witters, H.E., 2009. Development of a screening assay to identify teratogenic and embryotoxic chemicals using the zebrafish embryo. Reprod. Toxicol. 28, 308-320. http://dx.doi.org/10.1016/j. reprotox.2009.05.004.

Selderslaghs, I.W.T., Blust, R., Witters, H.E., 2012. Feasibility study of the zebrafish assay as an alternative method to screen for developmental toxicity and embryotoxicity using a training set of 27 compounds. Reprod. Toxicol. 33, 142-154. http://dx.doi. org/10.1016/j.reprotox.2011.08.003.

Sipes, N.S., Martin, M.T., Reif, D.M., Kleinstreuer, N.C., Judson, R.S., Singh, A.V., Chandler, K.J., Dix, D.J., Kavlock, R.J., 2011. Predictive models of prenatal developmental toxicity from toxcast high-throughput screening data. Toxicol. Sci. 124, 109-127. http://dx.doi.org/10.1093/toxsci/kfr220.

Stewart, A.M., Braubach, O., Spitsbergen, J., Gerlai, R., Kalueff, A.V., 2014. Zebrafish models for translational neuroscience research: from tank to bedside. Trends Neurosci. 37, 264-278. http://dx.doi.org/10.1016/j.tins.2014.02.011.

Sulston, J.E., Horvitz, H.R., 1977. Post-embryonic cell lineages of the nematode, Caenorhabditis elegans. Dev. Biol. 56 (1), 110-156.

Sulston, J.E., Schierenberg, E., White, J.G., Thomson, J.N., 1983. The embryonic cell lineage of the nematode Caenorhabditis elegans. Dev. Biol. 100 (1), 64-119.

The Zebrafish Model Organism Database [WWW Document], n.d. (Univ. Oregon, Eugene, OR 97403-5274. URL www.zfin.org). 